\title{
Earth Observation-Supported Service Platform for the Development and Provision of Thematic Information on the Built Environment - the TEP-Urban Project
}

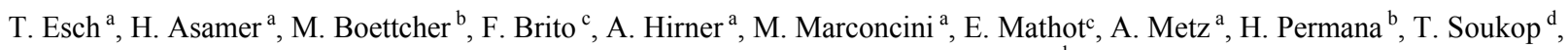 \\ F. Stanek ${ }^{\mathrm{e}}$, S. Kuchar ${ }^{\mathrm{e}}$, J. Zeidler ${ }^{\mathrm{a}}$, J. Balhar ${ }^{\mathrm{d}}$

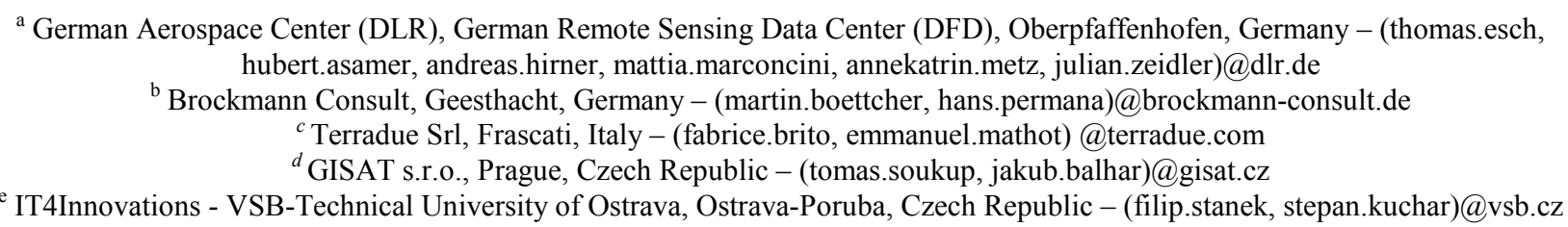

Commission VI, WG VI/4

KEY WORDS: Service platform, participatory, urban, monitoring, earth observation, decision support

\begin{abstract}
:
The Sentinel fleet will provide a so-far unique coverage with Earth observation data and therewith new opportunities for the implementation of methodologies to generate innovative geo-information products and services. It is here where the TEP Urban project is supposed to initiate a step change by providing an open and participatory platform based on modern ICT technologies and services that enables any interested user to easily exploit Earth observation data pools, in particular those of the Sentinel missions, and derive thematic information on the status and development of the built environment from these data. Key component of TEP Urban project is the implementation of a web-based platform employing distributed high-level computing infrastructures and providing key functionalities for i) high-performance access to satellite imagery and derived thematic data, ii) modular and generic state-of-the art pre-processing, analysis, and visualization techniques, iii) customized development and dissemination of algorithms, products and services, and iv) networking and communication. This contribution introduces the main facts about the TEP Urban project, including a description of the general objectives, the platform systems design and functionalities, and the preliminary portfolio products and services available at the TEP Urban platform.
\end{abstract}

\section{INTRODUCTION}

A large-scale transformation has occurred on Earth, largely ignored in headline stories on the topic of global change - for some years now, the number of people living in urban areas has exceeded that of those living in rural regions. The trend towards urbanization shows no sign of abating. In particular, cities in Asia and Africa are expanding at a staggering pace. Megacities are springing up in a matter of years and urban sprawls are emerging, spreading across extensive swathes of landscapes that, until recently, had been untouched by development or used for agricultural production. Hence, the future is urban. Today, approximately 7.2 billion people inhabit Earth. By 2050, this number will have risen to nine billion, 70 percent of which will be living in cities (UN, 2016). As a result, urban areas will account for 90 percent of population growth, 80 percent of increased prosperity and around 60 percent of energy consumption. Therefore, urban conurbations will occupy a key role as centers of political, economic and cultural life. They will exemplify the future, defining how the coming generations will live and work.

However, an effective management of urban development requires knowledge. How have urban regions developed in the past, and how should they do so in future? How can the opportunities that urbanization presents be put to good use? To what extent do cities damage the environment by consuming land and resources, polluting the air and the water, or by reducing biodiversity? To what extent are cities threatened by natural hazards and climate change? The answering of these questions represents are central challenges that society will face over the coming decades.
Urbanization playing out across the world has regional roots, but it also comes with common drivers and causes. A global view is required to identify what they are. It is here that Earth observation (EO) can make a valuable contribution. It helps differentiate between urban and rural settlement forms and to introduce systems of categorization and delineation. Satellitebased geo-information $\mathrm{p}$ delivers a current and comprehensive image of the built environment, while at the same time documenting its changes over time (Potere et al. 2009; Seto, 2009; Esch et al., 2010; Gamba and Herold, 2009).

\section{TEP URBAN PROJECT}

\subsection{ESA Thematic Exploitation Platforms}

In 2014 the European Space Agency (ESA) has started the initiative "EO Exploitation Platforms (EPs)" that represents a set of research and development activities aiming at the creation of an ecosystem of interconnected "Thematic Exploitation Platforms (TEPs)". Basically, a TEP is a collaborative, virtual work environment with one coherent user interface that provides access to EO data and the tools, processors, and Information and Communication Technology (ICT) resources required to efficiently extract thematic geo-information from mass EO data sources. In the first phase, ending in 2017, TEPs will be implemented for the topics Coastal, Forestry, Hydrology, Geohazards, Polar, Urban, and Food Security. (ESA, 2016a). 


\subsection{Urban Thematic Exploitation Platform}

The growing volume of environmental data obtained from satellites, in particular the Sentinels, represents a great opportunity for research and applications related to sustainable urban development. However, the volume of data available also poses a major challenge for users trying to exploit the full potential of the mass data collected by the Sentinel or other EO missions. Looking at massive volumes of image data acquired by modern EO missions, the traditional way of mission ground segments delivering data products to users who perform their work locally and separately from others needs to be revised. It is here where "Urban Thematic Exploitation Platform (U-TEP)" will provide support to the users.

The U-TEP aims at bridging the gap between the mass data streams and archives of the Sentinel satellites, but also other sources such as the Copernicus Contributing and Third Party Missions or Landsat programme (ESA, 2016b; USGS, 2016), and the information needs of users involved in urban and environmental science, planning, and policy. Therefore, the UTEP will provide a web-based, open and participatory platform that enables any interested user to exploit EO data easily and with no data download complications, to generate thematic information - for instance on mushrooming cities, the impact of climate change on the built environment, or the erosion of biodiversity due to human disturbance. More information about the U-TEP project can be obtained from the project website via https://urban-tep.eo.esa.int. At the same time, the website represents the entry point to the portal of the U-TEP platform and its products and services.

The U-TEP project aims at opening up new opportunities to facilitate effective and efficient urban management and the safeguarding of livable cities by systematically exploring i) the unique EO capabilities in Europe in combination with the big data perspective arising from the constantly growing sources of geo-data, ii) the massive processing power provided by modern ICT-infrastructures and technologies, and the capabilities of participation and sharing of knowledge by using new media and ways of communication.

In this context, U-TEP is supposed to initiate step changes regarding remote processing (bringing users-to-the-data), enabling technology (facilitating large-scale exploitation and timeliness of mass data analyses), distribution of expertise by sharing of data and methods (increase of assets, fostering of innovation, supporting benchmarking), and the establishment of open, integrative, participatory, collaborative concepts (community stimulation, increasing outreach).

\subsection{Team and User Community}

The U-TEP platform is developed by a consortium led by the German Aerospace Center (DLR), and including Brockmann Consult GmbH (DE), Gisat s.r.o. (CZ), IT4Innovations (CZ), and Terradue Srl (IT). Terradue Srl are contributing with their expertise in developing a portal, IT4Innovations, Brockmann Consult $\mathrm{GmbH}$, and DLR are together responsible for the data processing and thematic analyses, also including the provision of high performance computing processing infrastructures, services and data storage. The visualisation and WebGIS components are provided by Gisat s.r.o.. Associated partners of the U-TEP activity are CloudEO AG, GIM nv/sa, Indra Sistemas S.A., KTH Royal Institute of Technology, National Observatory of Athens, Pallas Ludens, Planetek Italia, SAP Deutschland SE \& Co. KG, and Ticinum Aerospace.

Regarding the user community of U-TEP, this initiative is designed to serve a broad spectrum of stakeholders and protagonists operating from global to local scale and including scientific communities, public authorities and governmental organizations, non-governmental and non-institutional communities, and the commercial sector. The main users already actively involved in the U-TEP project are the World Bank Group, the Group on Earth Observation, the European Environment Agency, the European Commission Directorate for Regional and Urban Policy, the International Society of City and Regional Planners, and the City of Prague.

\section{TEP URBAN PLATFORM AND SERVICES}

\subsection{Technical Concept}

The U-TEP technical approach is based on a generic, modular, multi-purpose systems design, facilitating maximum flexibility with respect to the adaptation to and integration of user requirements, application scenarios, processing and analysis technologies, and IT infrastructures. Thereby, U-TEP can be considered as an evolving framework platform, which brings users and functionalities closer to big data inventories, supports large-scale and complex data exploitation, and facilitates the sharing of data, technology, and knowledge. Therefore, the key components of U-TEP include:

1. Web-based, modular platform employing generic, distributed high-level computing infrastructures,

2. High-performance access to EO data and other sources of data relevant to urban analyses,

3. State-of-the art pre-processing, analysis, and visualisation techniques,

4. Customising functionalities for transfer of algorithms/products into services;

5. Functionalities to disseminate data, functionalities, and information, and

6. Support of networking and communication activities.

With that the U-TEP platform includes models for Platform as a Service (PaaS), Infrastructure as a Serviced (IaaS), Information as a Service (InaaS), and Software as a Service (SaaS). Here it is important to mention that the U-TEP platform is not designed to provide direct access to EO data and therewith covering functionalities of a classical data distribution tool. A schematic view of the basic systems components of U-TEP is presented in Figure 1.

In order to assure sustainable developments, U-TEP follows an Open Source strategy committing that the platform is based on Open Source components and modules, with an online presence at https://github.com/urban-tep. Moreover, the interfaces between the integrated software APIs are based on open standard specifications (e.g., OCCI, OGC).

\subsection{Basic Use Scenarios}

The systems design and functionalities of U-TEP platform are focused on the support of four basic use scenarios:

\section{Explore existing thematic content}

The user is enabled to discover and select a broad spectrum of existing thematic content on manifold urban themes provided on the U-TEP platform. Moreover, the user can upload own data and content (e.g., statistics) and jointly analyze and visualize the available thematic layers based on state-of-the-art tools of Geographic Information Systems and visualization. Any openly available thematic content or generated result can be exported. 


\section{Task individual on-demand analyses}

The U-TEP platform offers the possibility to define and conduct on-demand processing and production of thematic content based on multi-mission remote sensing imagery (in particular ESA missions including the Sentinel suite and Landsat imagery) and additional data sources (e.g., cadastral data, statistics, socioeconomic data). The additional data might either be publically available on the platform or individually uploaded by the user. For the processing and data analysis the U-TEP platform provides high-level processing solutions, state-of-the-art algorithms and high-performance IT-infrastructures supporting large-scale and near-real-time data exploitation. These activities and functionalities are partially free and open, while others are provided on a pay-per-use basis.

Develop, deploy and offer your own content or application To foster innovation and collaboration, the U-TEP platform allows for the individual development and deployment of analysis techniques, thematic products and application-oriented services. Therefore, the platform offers interfaces to upload and run user-defined algorithms and processes and provides functionalities to develop own methods and products (e.g., by using the Sentinel tool boxes) directly on the platform. Developed applications or products can then be presented and shared at the U-TEP platform application and product store.

\section{Learn more about innovative data sets and methods}

The U-TEP platform provides various opportunities to inform about innovative products, applications and initiatives and the latest state-of-the-art methods and solutions. These approaches and products might even be available for first testing on the platform by the interested user and the user can also contribute a rating of or feedback to the method or solution in order to support further optimization and/or operationalization.

\subsection{Data, Products and Services}

In the first phase of the TEP Urban project, a defined number of thematic value-adding (VA) processors will be implemented and operated on the platform. The focus is set on demonstrating functionalities to provide value-added basic EO data and innovative urban thematic geo-information products. The products and service portfolio of U-TEP scheduled for the first phase includes:

1. High-performance access to Sentinel-1, Sentinel-2, Landsat, and other selected EO missions,

2. On-demand generation of cloudless multi-temporal image stacks/mosaics,

3. On-demand generation temporal statistics indices,

4. Provision of global high resolution human settlements map,

5. On-demand generation of settlements masks,

6. Provision of global inventory on human settlements properties and patterns,

7. On-demand generation of regional maps of urban growth,

8. On-demand generation of regional imperviousness/greenness maps,

9. On-demand generation of regional maps of urban and peri-urban land use / land cover.

10. Functional service for development and deployment of new services and/or products by users,

11. Functional service for combination, analysis and visualization of data and products.

Figure 2 illustrates an example of the functional service for analysing and visualizing generated results. In addition, innovative technologies, services and products will be tested and demonstrated in the context of U-TEP, including crowdsourcing and the analysis of geo-located data from cell phones, transport systems, and/or social media (see Fig. 3)

\section{CONCLUSIONS AND FUTURE PERSPECTIVE}

It is the objective of the U-TEP project to establish a web-based platform for fast and convenient access, exploration, generation, analysis and visualization of EO imagery and other geospatial data and products related to the built environment. Its thematic value-adding (VA) processors will provide on-demand and upto-date geo-information about the status and development of urban areas for any region and time of the user's interest. Therefore, U-TEP will be integrated in an infrastructure for very fast, cost-effective and operational access to multiple satellite mission archives (in particular ESA and Sentinel archives), which is built on expert as well as user knowledge and requirements. Currently, most activities for thematic information retrieval are non-generic and often short term solutions as they are mainly project funded or following a very specific focus (e.g., of selected user community). Depending on the resources, individual processors and product solutions are developed and set-up. Moreover, data is collected or purchased from various sources. Most urban-related data and geoinformation depends on local city-based sources that are limited to administrative boundaries. Such information layers are not harmonized and do not allow comparability on (inter)national level. Frequently, the data collection practices are based on sectorial approaches with limited integrated views. Numerous datasets available are then disconnected and difficult to integrate.

Here, the technological progress that U-TEP is expected to initiate, will provide a number of advantages for the users. Amongst others, these include:

1. Free and open access to and use of state-of-the art infrastructures (computing, data management, storage), techniques (e.g., multi-source data fusion and analysis) and EO-derived information products.

2. Efficient exploitation of full spectrum of available EO data streams and archives.

3. Validated and benchmarked algorithms and products.

4. Access to network of experts and stakeholders that share experiences and best practice applications.

5. Market place of ideas and driver of innovation;

6. Seed point for the animation of new user communities outside EO/geo-sector.

7. Gaining of better knowledge on urban system and increased efficiency, effectiveness and sustainability of functions and services in policy, planning, economy, and science.

These opportunities have already found their way into almost any part of our ever-day-life and a targeted effort to exploit the capabilities of the information technologies and services for a sustainable future development of the "human habitat" appears quite promising.

\section{ACKNOWLEDGEMENTS}

The authors would like to thank the European Space Agency (ESA) for their funding of the project "Urban Exploitation Platform" (ESA Contract No. 4000113707/15/I-NB). 


\section{REFERENCES}

ESA, 2016a. European Space Agency, Website about Thematic Exploitation Platforms (TEP). https://tep.eo.esa.int (14. Apr. 2016).

ESA, 2016b. European Space Agency, Website about Copernicus Programme and Services. http://www.esa.int/Our_Activities/Observing_the_Earth/Copern icus/Overview. (14. Apr. 2016).

Esch, T., Taubenboeck, H., Heldens, W., Thiel, M., Wurm, M., \& Dech, S. (2010). Urban remote sensing e how can earth observation support the sustainable development of urban environments?. In Proceedings of 46th ISOCARP Congress, 1923 September 2010, Nairobi, Kenya.

Gamba, P., \& Herold, M. (2009). Global mapping of human settlement e Experiences, datasets, and prospects. CRC Press.

Seto, K. C. (2009). Global urban issues e a primer. In P. Gamba, \& M. Herold (Eds.), Global mapping of human settlements e Experiences, datasets, and prospects. Boca Raton: Taylor \& Francis Group.

UN, 2016. 2014 Revision of World Urbanization Prospects of the Population Division of the Department of Economic and Social Affairs of the United Nations. http://esa.un.org/unpd/wup/ (14. Apr. 2016).

USGS, 2016. United States Geological Survey, Website about Landsat Missions. http://landsat.usgs.gov (14. Apr. 2016). 
The International Archives of the Photogrammetry, Remote Sensing and Spatial Information Sciences, Volume XLI-B8, 2016 XXIII ISPRS Congress, 12-19 July 2016, Prague, Czech Republic

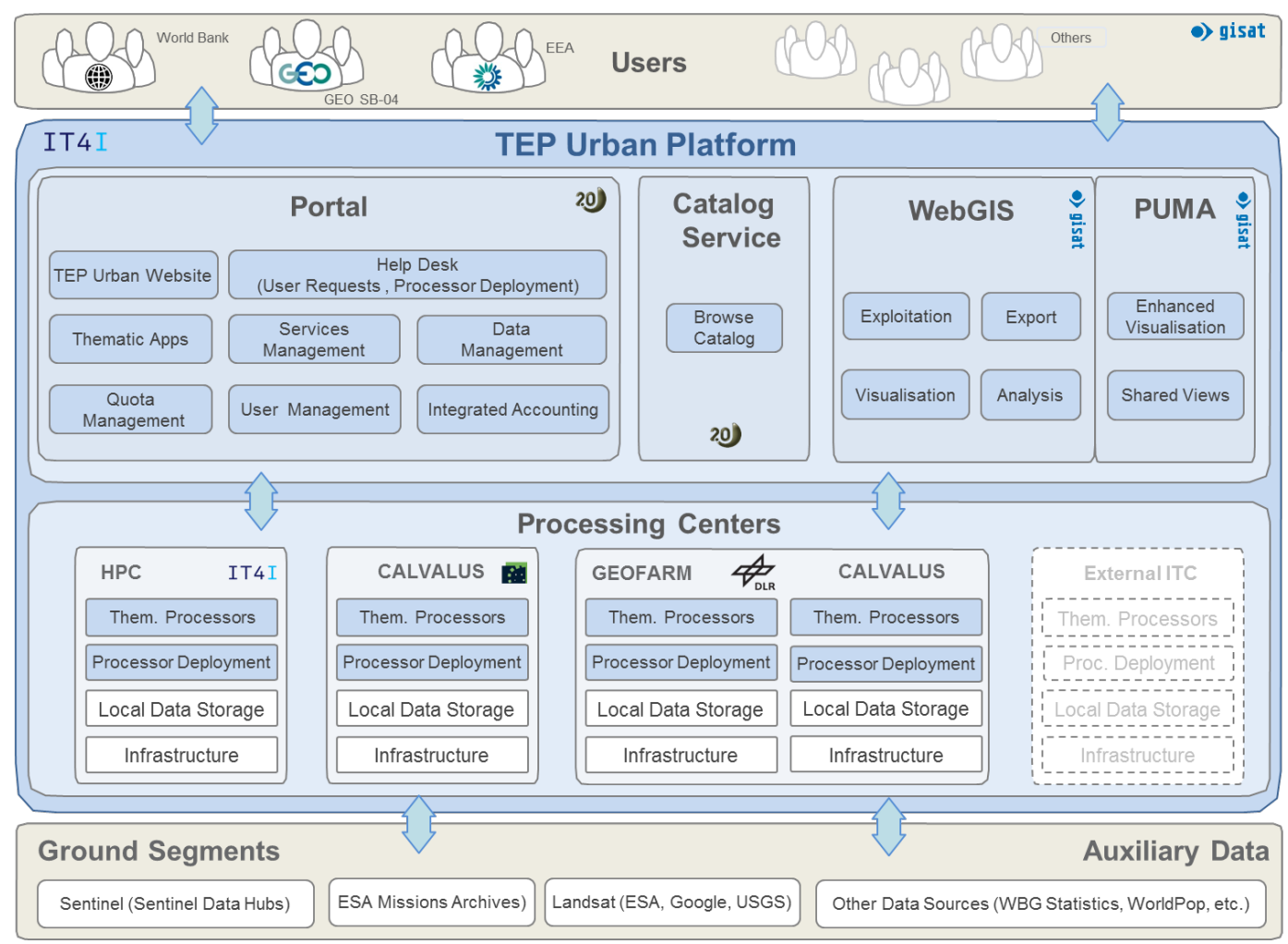

Figure 1. Schematic view of basic components of the Urban Thematic Exploitation Platform (U-TEP)

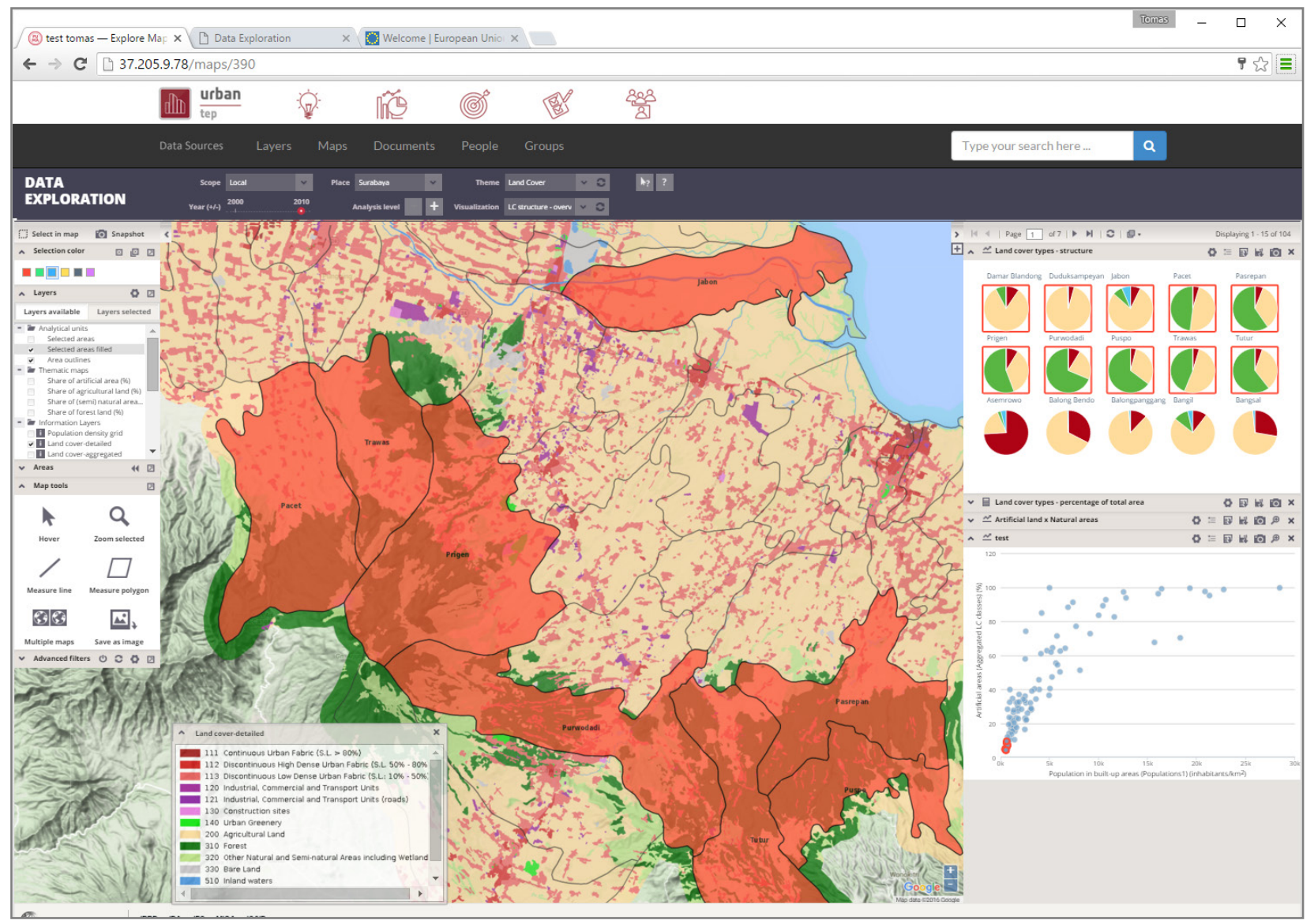

Figure 2. Interface of U-TEP service for customized analysis and visualization of data and products. 
The International Archives of the Photogrammetry, Remote Sensing and Spatial Information Sciences, Volume XLI-B8, 2016 XXIII ISPRS Congress, 12-19 July 2016, Prague, Czech Republic

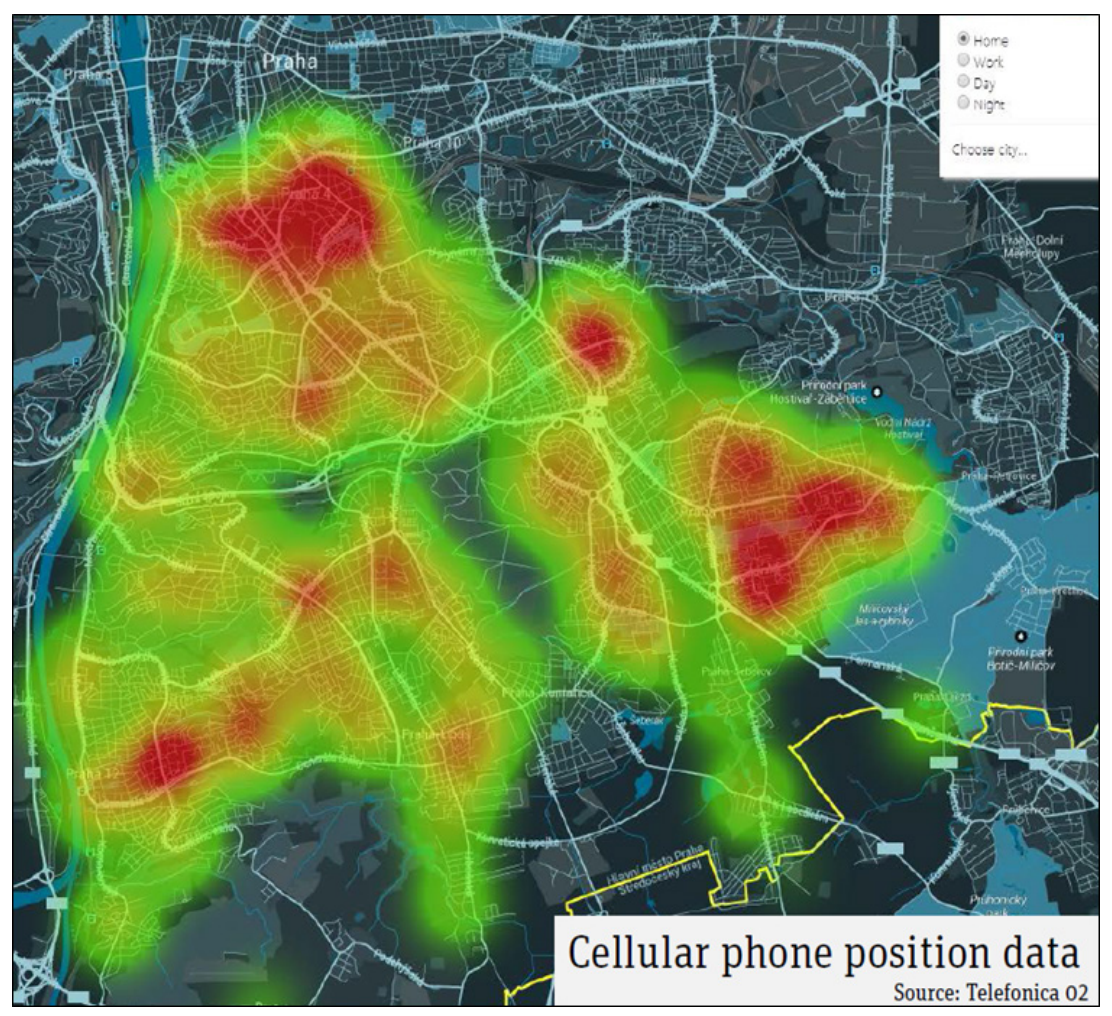

Figure 3. Example of geo-information product derived from near-real-time mobility service based on cell phone data. 